Model of evaluation and Modelo de evaluación certification of askills $\mathbf{y}$ certificación de workshop integrating integrado de tecnología y technology and processes. procesos

Pilot study for skills

formation in construction

engineering at UCN

\section{Experiencia piloto}

en la formación por

competencia en ingeniería

en construcción UCN

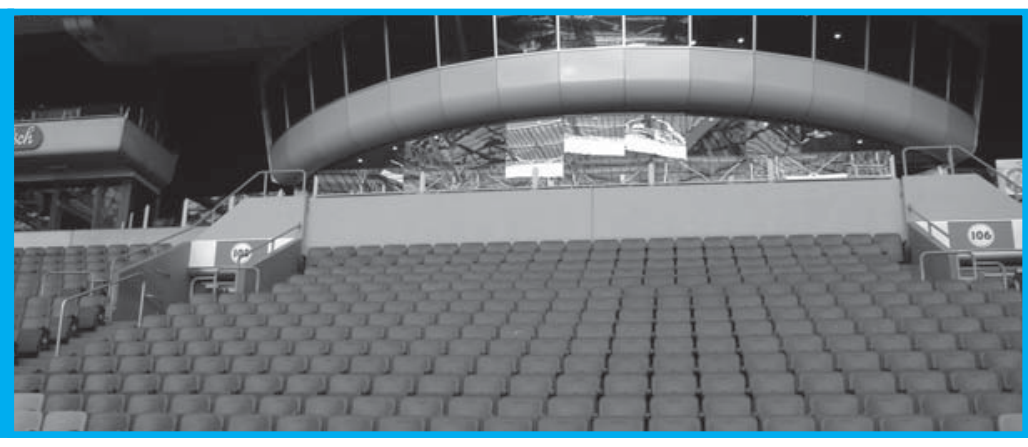

Autores

GONZÁLEZ LEÓN, A. agonzale@ucn.cl

GONZÁLEZ CHANG, C.

Universidad Católica del Norte cargonzalez@ucn.cl Antofagasta, Chile

Fecha de recepción

Fecha de aceptación
02/01/2013

21/03/2013 
Resumen El artículo expone una experiencia piloto de implementación de un modelo de evaluación y certificación de competencias en el ámbito universitario, particularmente en la carrera de Ingeniería en Construcción de la Universidad Católica del Norte. Se expone el proceso de construcción de la norma de competencia y el diseño y validación de los instrumentos de evaluación.
Además se exponen los principales resultados relacionados con rendimiento y satisfacción de los estudiantes. Los resultados muestran que el modelo de evaluación tiene un efecto significativo en el aprendizaje y desempeño académico de los estudiantes.

\section{Abstract}

The article describes a pilot implementation of a model evaluation and certification of skills in the university environment, particularly in the career of Construction Engineering at the Catholic University of the North. We describe the construction process of the standard and the design and validation of assessment instruments. It also presents the main findings related to performance and student satisfaction. The results show that the evaluation model has a significant effect on learning and student achievement

Keywords: Competence, Evaluation, Certification, Higher Education. 


\section{Marco conceptual}

\subsection{Introducción}

La carrera Ingeniería en Construcción, en su constante búsqueda de la mejora de sus procesos educativos y de innovación metodológica de la enseñanza-aprendizaje, decide dar un paso más a través de la incorporación de la formación por competencia en algunas de sus asignaturas. Para ello, aborda la tarea de incorporar esta innovación metodológica, en los cursos integradores que están incorporados en la malla curricular de la carrera, como son el Taller Integrado de Tecnología y Procesos; las prácticas preprofesionales y el Taller Integrado de Administración de Proyectos. El presente trabajo abordará la innovación metodológica del Taller Integrado de Tecnología y Procesos. Este se ubica en la mitad de la malla curricular y tiene la particularidad de integrar varias asignaturas previas y sirve además como control de calidad del avance curricular de los estudiantes, en consecuencia es una buena etapa para evaluar a los estudiantes, si han alcanzado algunas de las competencias declaradas en el perfil de egreso.

El "Taller Integrado de Tecnologías y Procesos", está vinculado al Dominio I del Perfil de Egreso de la Carrera y en consecuencia debería hacerse cargo de desarrollar cuatro áreas de competencia, a saber: evaluar el proyecto de construcción y su entorno; planificar

El Perfil de Egreso de Ingeniería
en Construcción contempla tres
ámbitos de acción (Dominios
de Competencia):
- Ingeniería de Procesos de
Construcción
- Dirección y Gestión de
Proyectos de Construcción
- Dirección y Administración
de empresas del sector
construcción
operacionalmente los procesos y recursos para la materialización del proyecto de construcción y de mantenimiento; materializar los procesos productivos del proyecto de construcción y de mantenimiento y solucionar sistemas de instalaciones domiciliarias. Consecuente con lo anterior, los académicos a cargo del taller, asumen el desafío de diseñar, implementar y evaluar una metodología de evaluación y certificación de competencias, aprovechando las fortalezas, ya existentes en el curso producto de la constante revisión, mejoramiento e implementación de innovaciones metodológicas, como son: trabajo en equipo con proyectos reales; la incorporación de profesionales destacados en ámbito externos como profesores de la asignatura; seguimiento de obras de construcción reales e interactuando con las jefaturas de las mismas, todo con la finalidad de facilitar y medir con mayor objetividad el desarrollo de competencias de los es- tudiantes. Es importante señalar que, a pesar de los cambios metodológicos introducidos en este taller, persistían dificultades a la hora de evaluar el aprendizaje de los estudiantes, principalmente en relación al grado de subjetividad de las evaluaciones realizadas, falta de claridad respecto de las competencias requeridas por los empleadores y desconocimiento de los parámetros para evaluar la adquisición de competencias en los estudiantes.

\subsection{Evaluación por competencia}

El proyecto educativo de la Universidad Católica del Norte ha establecido como modelo de formación la educación basada en el desarrollo de competencias, entendiendo este como uno de los enfoques que muestra mayor coherencia con uno de sus pilares fundamentales que apunta hacia la educación centrada en el aprendizaje. Este enfoque facilita la vinculación entre los procesos de formación y el mundo del trabajo, por cuanto permite la flexibilidad necesaria para estar en constante mejora y actualización respecto de referentes tanto internos como externos. Además, otra de sus características radica en responder a la evaluación de la calidad de la formación, a través de procesos de acreditación o certificación centrados tanto en resultados como en procesos. Es así que en este nuevo enfoque la evaluación no solo se centra en el manejo de contenidos ni de saberes conceptuales, sino que también considera la evaluación en relación a la presencia de una serie de atributos relacionados con el conocimiento y sus aplicaciones, actitudes, valores, etc. y la descripción acerca del nivel en el cual el estudiantes es capaz de desempeñarse en una determinada situación (González y Wagenaar, 2003).

Por otro lado, la Evaluación de Competencias es definida como la Evaluación de Logros (Whitear, 1995). El propósito de la evaluación consiste en realizar juicios, con un grado razonable de objetividad, acerca de si se han alcanzado o no el desarrollo de ciertas competencias e identificar aquellas áreas de desempeño que requieren ser fortalecidas.

Como todo proceso de evaluación pedagógica, la evaluación por competencias debe cumplir con dos finalidades principales (Sanmartí, 2007). En primer lugar debe tener un carácter social, ya que debe estar orientada a constatar, ante los estudiantes, y la sociedad en general, y particularmente al empleador, el nivel de determinadas competencias al finalizar una determinada etapa, y en segundo, tiene un carácter pedagógico, orientado a identificar los cambios a introducir, tanto en el proceso de enseñanza, por parte del profesor, como en el aprendizaje, por parte del estudiante. 


\subsection{Certificación}

Ahora bien, la evaluación por competencias nos presenta el desafío de incorporar a la evaluación el proceso de certificación. El término certificación de competencias es utilizado en el ámbito laboral, para determinar la presencia o ausencia de una determinada competencia, ya sea para evaluar el desempeño, seleccionar y/o promover a un integrante de la organización.

Para la realización de este proceso, se utiliza la Norma de Competencia, la cual debe ser construida entre la institución formadora y el sector productivo. Esto permite la adecuada orientación del proceso formativo a las competencias requeridas, por parte de la institución formadora, y por otro, asegura al futuro empleador de la presencia de la competencia en el profesional. Para lograr comprender mejor esta idea, repasaremos algunos conceptos definidos por Catalano, A., et al. (2004).

Certificación de Competencias: Reconocimiento formal de las competencias demostradas por un individuo a partir de un proceso de evaluación realizado sobre la base de normas de competencias establecidas por una empresa para un rol laboral determinado, o consensuadas por los actores de un sector de actividad.

Evaluación de competencias: Es el proceso por el cual un evaluador reúne signos de evidencia de la competencia que un integrante de la organización debe demostrar para el ejercicio de un determinado rol laboral. El piso de los signos de evidencia que el evaluador utiliza, está enunciado en la norma de competencia, de la cual deben ser tomados los signos de evidencia para elaborar los instrumentos de evaluación.

Norma de competencia: Es la especificación de un desempeño laboral que ha sido acordado entre los actores del mundo del trabajo para ser utilizado en procesos de selección y/o de formación profesional, y/o de evaluación, y/o de desarrollo de carrera, y/o de certificación. Su utilización en cada uno de estos procesos debe ser acordada previamente entre los actores.

Normalización de competencias: Proceso mediante el cual los estándares de competencia construidos son validados, rectificados y ratificados por los actores del mundo del trabajo del sector, y convertidos en norma de competencia. En ese consenso se determina la aplicación de la norma a diferentes procesos: de selección, de formación, de proyección de carrera profesional, o de certificación de trabajadores.

En un contexto educativo, como es el estudio de este caso, para ser juzgado como competente, se efectuará un uso pedagógico de los instrumentos de certifica- ción laboral, como lo es la Norma de Competencia. En consecuencia, los estudiantes deberán demostrar en los ambientes posibles de recrear pedagógicamente, sus habilidades para desempeñar roles específicos de acuerdo a ciertos referentes estandarizados. Este referente fue construido sobre la base de aportes recibidos por profesionales expertos del mundo laboral, la opinión de los egresados y los académicos que participan en el Taller. De esta forma, es posible acercarse a medir el logro alcanzado de la competencia "certificación", mediante instancias de evaluaciones objetivas de las competencias, las que permiten aproximarse, en el transcurso del proceso formativo, al nivel de avance con respecto al logro del perfil de egreso, instancia final del profesional Ingeniero Constructor.

\section{Diseño}

\subsection{Objetivos}

- Favorecer el aprendizaje de los estudiantes a través del uso de estrategias de evaluación pertinentes.

- Facilitar la puesta en práctica de las competencias desarrolladas por los estudiantes, vinculados al Dominio I del Perfil de Egreso.

- Diseñar una metodología de evaluación de competencias que permita su transferencia a otras carreras o espacios de integración

\subsection{Modelo conceptual}

El proceso de evaluación y certificación de competencias desarrolladas en este trabajo fue estipulado en seis etapas sucesivas que son descritas a continuación y que están graficadas en la figura $N^{\circ} 1$.

La primera etapa corresponde a la definición de la Norma de Competencia, la que es tomada del perfil de egreso, considerando los dominios de competencia sobre los cuales se hará cargo el Taller en estudio. Luego se proponen y definen cuáles serán los momentos más convenientes y sobre qué se producirán las evaluaciones. Definido lo anterior, se construye el Instrumentos de evaluación, con sus respectivos entregables y niveles de gradualidades que puedan alcanzar los estudiantes. Enseguida y frente a una comisión integrada por los académicos que componen el taller más profesores invitados que reciben el producto de este proceso, los estudiantes son evaluados y finalmente calificados.

Para lograr lo descrito, fue necesario planificar y diseñar todo el proceso, el que estuvo a cargo de una comisión compuesta por los académicos responsables del curso-taller y dos asesores pertenecientes al Centro de Innovación Metodológica y Tecnológica - CIMET 
de la Universidad. El trabajo en comisión estuvo compuesto por tres fases.

La primera fase tiene como referente el análisis de la Norma de Competencia, en la que se define qué, cuándo y dónde evaluar, a la vez que se establece el rol de cada uno de los actores que intervienen en el proceso de evaluación: docentes, estudiantes y asesores. El equipo de docentes y asesores elaboró una agenda de trabajo en la que se estipularon claramente las etapas del proceso, y los compromisos y tareas a desarrollar por cada uno de los miembros.

En la segunda fase se elaboró una matriz de análisis para cada una de las competencias, en la que se indica la competencia a evaluar, los estándares de calidad que las regulan, y los indicadores que evidencian el grado de desarrollo de dicha competencia por parte de los estudiantes, y que constituirán los indicadores de logro de dichas competencias.

En la tercera fase, se definen los instrumentos de evaluación. A partir de los indicadores de logro generados, se decidió la utilización de Tablas de Especificación de Criterios de Evaluación (Rúbrica) como instrumento de evaluación, ya que este responde a la necesidad de comunicar a los estudiantes con toda claridad los resultados de aprendizaje y, muy particularmente, la necesidad de hacer explícitos desde un principio cuáles son los criterios a evaluar y cómo se calificarán los resultados esperados. Posteriormente se establecieron los niveles de desempeño (Destacado, Competente, Debe Mejorar e insatisfactorio) y su conversión a una nota o calificación, el tipo de evaluación que se realizaría, el tiempo de aplicación de la evaluación y el momento en el que se entregaría a los estudiantes las rúbricas con los criterios de evaluación para orientar de mejor manera el proceso de aprendizaje.

\subsection{Metodología de Implementación}

La implementación de la metodología abarcó varios aspectos a saber, entre los cuales se destacan los siguientes:

a) Presentación de la actividad: fue realizada por el equipo de la innovación metodológica, compuesta por los académicos del taller acompañados por las asesoras del CIMET, ello fue realizado en el primer día del Taller, ante todo el grupo curso. Se apoyo con una presentación resumida de la metodología a implementar con la intervención de cada uno de los exponentes y la posibilidad de que los estudiantes consultaran.

b) La metodología y sus instrumentos: se explicaron cada una de las etapas desarrolladas para lograr la metodología a implementar, junto con explicar cada uno de los instrumentos que esta metodología conlleva, los tiempos y momentos de evaluación y cómo se les evaluaría. Los beneficios y problemática que ello involucraría, dado que habría mucho trabajo en equipo, evaluación de los avances, entregas parciales de un proyecto de edificación de dos pisos. Además, de trabajo en terreno con mediciones de productividad de la mano de obra y planificación operacional con las cuadrillas de obras.

c) El trabajo a realizar: los estudiantes deben desarrollar dos trabajos en paralelo, uno en terreno directamen-

Figura 1. Modelo del Proceso de normalización, evaluación y certificación de competencias

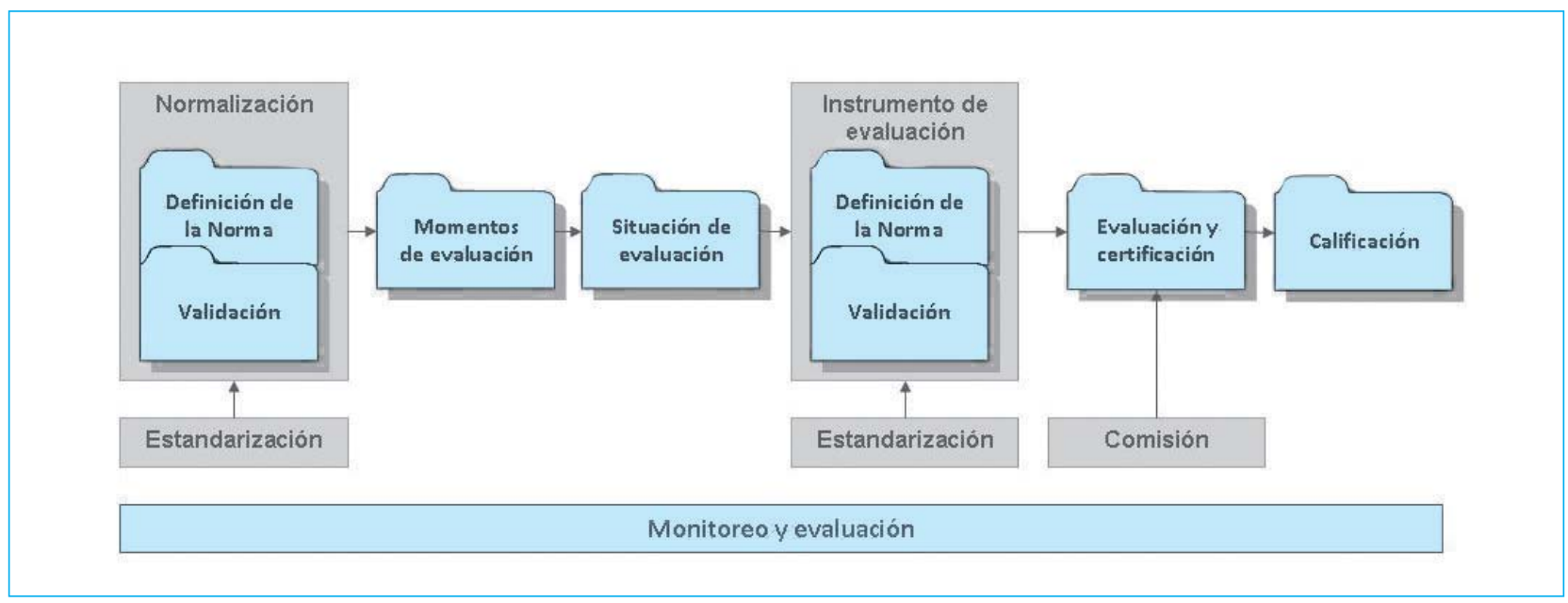


te, durante un mes, midiendo productividad de la mano de obra en diferentes etapas que se encuentre la construcción y planificando operacionalmente en conjunto con los supervisores de primera línea, distintos procesos constructivos, analizando recursos materiales, humanos, secuencias y flujoramas; midiendo su grado de cumplimiento semanal. Por otro lado, deben desarrollar un proyecto constructivo consistente en una vivienda unifamiliar de máximo dos pisos, proponiendo materialidad, soluciones constructivas, especificaciones técnicas, cubicaciones y recursos a utilizar, bajo determinadas condiciones de suelo, topografía y clima. Para su realización cuentan con un tiempo de cien días. Ambos trabajos deben ser evaluados por una comisión compuesta por todos los profesores del Taller, la primera evaluación se realiza a la mitad del semestre y la segunda al final de él. En los dos trabajos, los estudiantes tienen que desarrollar entregables, compuesto por un informe escrito, que recaba resultados, deficiencias detectadas, soluciones, propuestas de mejoramiento y conclusiones de las obras de construcción visitadas por un lado y en el otro trabajo, entregando el proyecto de edificación de la vivienda con los planos de planta, cortes, elevaciones, instalaciones detalles y soluciones constructivas; especificaciones técnicas; cubicaciones, propuesta de recursos y gráfica con los flujoramas entre otros.

d) Instancias de retroalimentación: durante el desarrollo de los trabajos, existen instancias de retroalimentación permanente, con los propios profesores y ayudantes del Taller, los encargados de las obras y otros profesores de la unidad. Lo anterior se realiza tanto antes de las presentaciones formales como luego de ellas.

e) Las Evaluaciones: la situación de evaluación del Taller Integrado de Tecnologías y Procesos se lleva a cabo en dos etapas, una en la mitad del proceso, mediante la evaluación del proceso realizado en las obras de construcción visitadas y la otra al final de la secuencia didáctica del curso con la presentación del proyecto de edificación. En ambas es mediante la presentación grupal de forma oral con soporte audiovisual frente a sus pares y a una comisión compuesta por los profesores del Taller y otros profesores invitados de cursos superiores. La comisión somete a los expositores a preguntas principalmente referidas a los aspectos técnicos del proyecto pero, sin dejar de lado otros aspectos tales como, dicción, desplante, seguridad y conocimiento del tema. Cada grupo está compuesto entre 4 y 6 estudiantes como máximo y exponen en un tiempo de 30 minutos. La situación de evaluación fue registrada mediante audio y video para una posterior retroalimentación

\section{Resultados}

Se presentan a continuación los resultados más relevantes luego de tres años de implementación (2008, 2009 y 2010) de esta innovación metodológica. Específicamente se analizan las tasas de Aprobación de los estudiantes, el Rendimiento Académico y la Satisfacción con la innovación implementada. Para este último aspecto se le aplicó a los estudiantes, al término del taller, un cuestionario tipo Likert con cinco alternativas de respuestas (desde Totalmente en desacuerdo (1) a Totalmente de acuerdo (5)), donde se les pedía valoraran la innovación en términos de su coherencia y organización, su contribución al aprendizaje, los procedimientos de evaluación y seguimiento, y la claridad de los criterios de evaluación.

\subsection{Estadística de rendimiento y aprobación}

En lo que respecta a Rendimiento y Aprobación, los resultados del primer año de implementación de esta metodología de evaluación de competencias, dan cuenta de un cambio positivo en la tendencia del porcentaje de aprobación de esta asignatura, aumentando significativamente la proporción de estudiantes aprobados, llegando en el año 2010 a la totalidad de los alumnos que cursaban este taller (ver gráfico $N^{\circ} 1$ ). De igual modo, resulta relevante destacar la disminución progresiva de la proporción de estudiantes que deben rendir examen recuperativo, la cual alcanza en los dos últimos años su reducción total.

Por otra parte, se advierte una mejora sustancial en las calificaciones de los estudiantes, principalmente en los que respecta al promedio de notas de cátedra y el promedio de notas final del taller (ver gráfico $\mathrm{N}^{\circ}$ 2). En conjunto, estos resultados dan cuenta que esta metodología mejora significativamente no solo los aprendizajes instrumentales, como es de suponer, sino también mejora los aprendizajes conceptuales.

Gráfico 1. Tasa de Aprobación

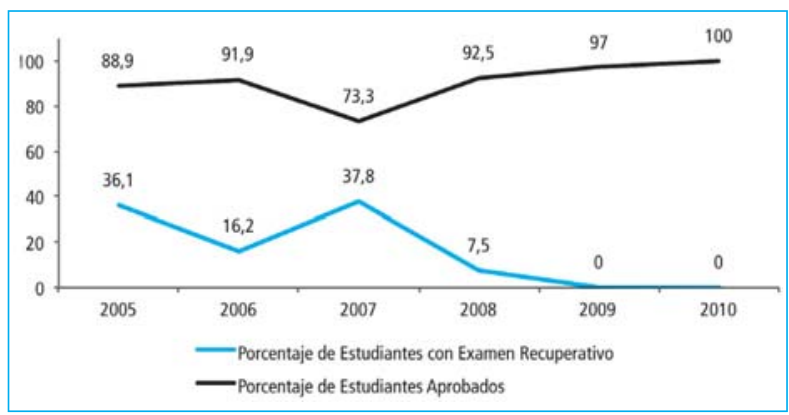


Gráfico 2. Rendimiento Académico

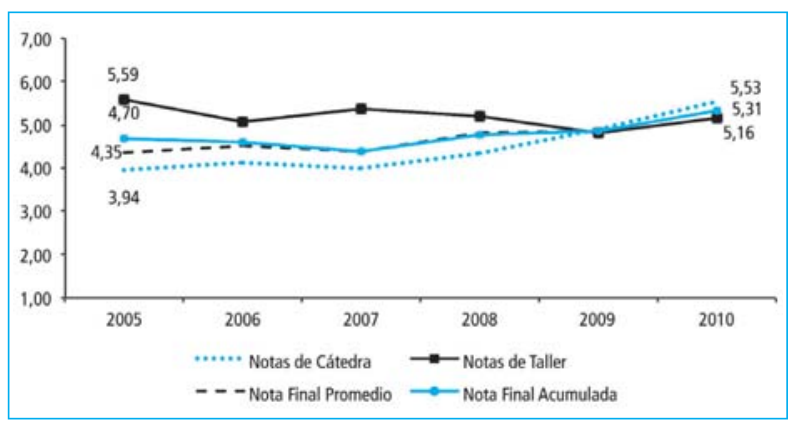

\subsection{Opinión y satisfacción de los estudiantes}

Las respuestas de los estudiantes al cuestionario aplicado, dan cuenta de una valoración positiva respecto de las dimensiones evaluadas, encontrando puntuaciones medias superiores al promedio teórico en los cuatro ámbitos medidos (3.5). En este sentido, la mayoría de los estudiantes consideran que existió una clara estructuración y organización de las actividades desarrolladas en el taller, así como también que dichas actividades permitieron alcanzar el logro de los aprendizajes esperados. En cuanto a la evaluación, los estudiantes mencionan que existieron instancias de retroalimentación y seguimiento por parte de los profesores, y que estaban informados respecto a los criterios con los que serían evaluados (ver gráfico $\mathrm{N}^{\circ} 3$ ).

Gráfico 3. Puntuaciones Medias por Dimensión

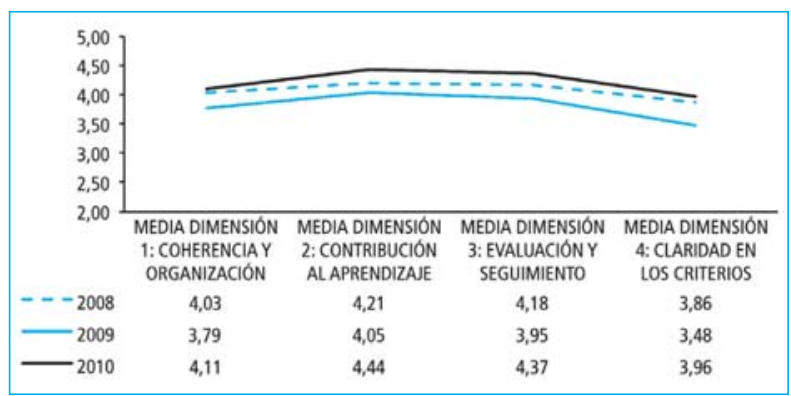

En cuanto al grado de Satisfacción de los Estudiantes, es posible advertir que, de acuerdo a su valoración, esta experiencia contribuyó significativamente a la adquisición de los aprendizajes del Taller Integrador I: "Tecnologías y Procesos", lo cual se refleja en el alto nivel de satisfacción que declaran los estudiantes al término de cada implementación, con una puntuación media superior a los 7 puntos en una escala de 1 a 10 (ver gráfico $\mathrm{N}^{\circ} 4$ ). Lo anterior se ve reforzado por el aumento en el porcentaje de estudiantes que consideran que esta metodología les ha permitido alcanzar aprendizaje más significativos en comparación con otras metodología experimentadas en su proceso de formación (ver gráfico N5).

Gráfico 4. Evaluación de Satisfacción

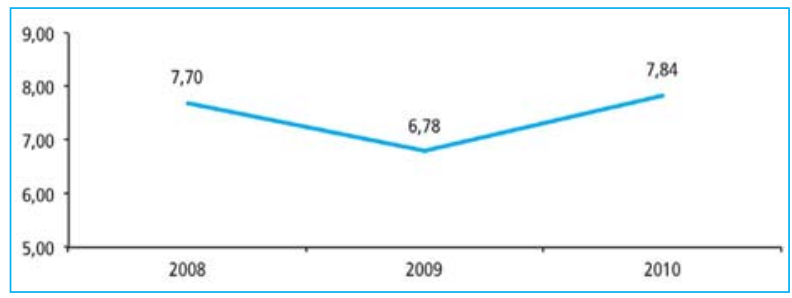

Gráfico 5. Valoración de los Aprendizaje Adquiridos

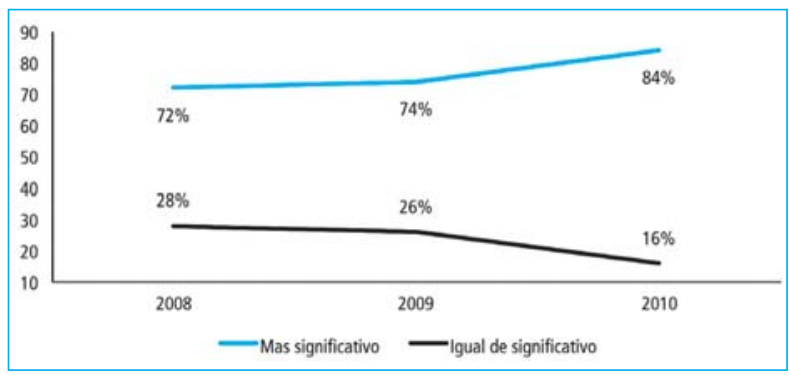

Teniendo estos datos en consideración, resulta bastante coherente que la mayoría de los estudiantes recomienden y prefieran la metodología utilizada en esta experiencia por sobre la metodología tradicional y que además, expresen que les gustaría trabajar en otros ramos con este tipo de metodología (ver gráfico $\mathrm{N}^{\circ} 5$ ).

Gráfico 6. Valoración de la Metodología utilizada

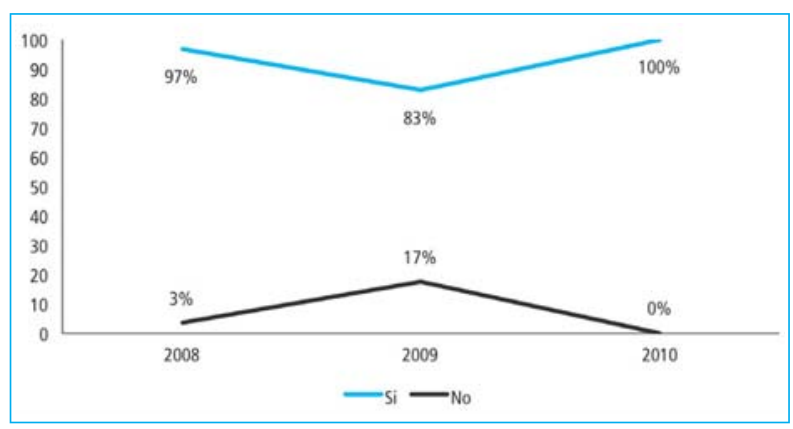




\subsection{Dificultades de la implementación}

- Puede resultar complejo, conseguir la colaboración sistemática de profesionales externos, debido a las exigencias propias de sus lugares de trabajo.

- El diseño de las actividades e instrumentos de evaluación, implicó flexibilizar los tiempos de trabajo, para adaptarse a los horarios de los profesionales externos.

- Resulta difícil, como docentes, en una primera implementación, centrarse en la retroalimentación y evaluación de desarrollo de competencias, más que en la calificación de los estudiantes. Para los estudiantes, es difícil adaptarse a trabajar siguiendo indicadores (definidos en los instrumentos de evaluación), en este aspecto surgieron algunas ansiedades y dificultades para comprender los instrumentos.

\section{Conclusiones y propuestas de mejoras}

El modelo de evaluación propuesto, resulta ser coherente con el modelo de formación por competencias y el Proyecto Educativo de la Universidad. Corresponde a una metodología que promueve una formación integral, subrayando la promoción y adquisición, no solo de conocimientos sino también de habilidades y actitudes, en el marco de un proceso en constante evaluación, actualización y adaptación activa y flexible a las demandas del entorno.

Asimismo, incorpora la formalización de procesos de evaluación y retroalimentación y de distintos agentes (docentes internos, externos, empleadores, estudiantes, egresados), bajo la lógica de evaluación en $360^{\circ}$, transformando la evaluación en un proceso participativo.

Junto con ello, la evaluación de competencias se convierte en un proceso de evaluación y retroalimentación para el currículo, entregando constante información valiosa para la toma de decisiones en relación al proceso formativo.

Con respecto al impacto en el aprendizaje de los estudiantes, este modelo exige que las situaciones de aprendizaje y evaluación sean lo más próximo a las situaciones a las cuales los estudiantes deberán enfren- tar en el mundo laboral, y en donde deberán desplegar sus aprendizajes. Esto se encuentra estrechamente relacionado con una mayor motivación e implicación del estudiante en su propio proceso formativo y con una mejora en la calidad de los aprendizajes. Quedando lo anterior refrendado por el alto grado de satisfacción mostrado por los estudiantes en la encuesta respectiva $(7,84$ sobre un máximo de 9,0$)$ y comparativamente mucho mayor respecto a la metodología tradicional (84\% frente a $16 \%)$.

Se considera entonces, que este proceso puede ser una propuesta concreta para la implementación de un modelo de evaluación integral, que promueve la vinculación con el medio, coherente con la formación por competencia y la educación centrada en el aprendizaje, donde el estudiante asume un rol más activo en este tipo de trabajos, contribuyendo a una evaluación más objetiva de la formación impartida, y por sobre todo aportando significativamente al mejoramiento del proceso de aprendizaje de los estudiantes. Asimismo, tal como se mencionó anteriormente, la presente propuesta, representa una aproximación pedagógica de la certificación de competencias aplicadas en el mundo laboral.

A la fecha, este sistema de evaluación se ha rutinizado dentro del curso, lo que ha favorecido su sistematización y mejora permanente, a la vez que se ha podido socializar la experiencia y perfeccionar el sistema evaluativo y las situaciones de evaluación propuestas. Asimismo, el proceso trae como consecuencia la mejora en otros procesos: incorporación de otro taller integrador, mejora de las prácticas profesionales, entre otros.

Desde su primera implementación (2008), se han mejorado los siguientes aspectos en las sucesivas implementaciones:

- Se incorporan más profesionales del ámbito laboral a través de la Validación de la Norma de Competencia e Instrumentos de Evaluación

- Se realiza inducción a estudiantes respecto al uso pedagógico de los instrumentos de evaluación.

- Los instrumentos son socializados con los estudiantes al inicio del semestre.

- Se mejora el instrumento de evaluación, para favorecer su comprensión por parte de estudiantes y facilitar la aplicación por parte de los docentes 


\section{Referencias Bibliográficas}

González, J. Y Wagenaar, R. (2003). Tuning Educational Structures in Europe. Informe Final. Fase Uno. Bilbao, España. Universidad de Deusto y Universidad de Groningen

Sanmartí, N. (2007). Evaluar Para Aprender. 10 Ideas Clave. Editorial GRAÓ. Barcelona.
Catalano, A. MA.; Avolio de Cols, S.; Sladogna, M. G. (2004) Diseño curricular basado en normas de competencia laboral: conceptos y orientaciones metodológicas: BID/ FOMIN; Cinterfor. Buenos Aires.

Whitear, G. (1997). Para entender la evaluación. Competencia laboral. Normalización, certificación, educación y capacitación. Antología de lecturas, tomo 1. México: Conocer y Alambra Mexicana. 